\title{
Upregulation of CCT3 Predicts Poor Prognosis and Promotes Cell Proliferation via Inhibition of Ferroptosis and Activation of EGFR Signaling in Lung Cancer
}

\section{Kun Wang}

Yunnan Kungang Hospital, Kunming Fourth People's Hospital, Seventh Affiliated Hospital of Dali University

Jian $\mathrm{He}$

Yunnan Kungang Hospital, Kunming Fourth People's Hospital, Seventh Affiliated Hospital of Dali University

\section{Changling Tu}

The Third Affiliated Hospital of Kunming Medical University

Hui Xu

Yunnan Kungang Hospital, Kunming Fourth People's Hospital, Seventh Affiliated Hospital of Dali University

\section{Xugang Zhang}

Yunnan Kungang Hospital, Kunming Fourth People's Hospital, Seventh Affiliated Hospital of Dali University

\section{Yongchang Lv}

Yunnan Kungang Hospital, Kunming Fourth People's Hospital, Seventh Affiliated Hospital of Dali University

Chao Song ( $\sim$ chaosong6870@163.com )

Yunnan Kungang Hospital, Kunming Fourth People's Hospital, Seventh Affiliated Hospital of Dali University

\section{Research Article}

Keywords: lung cancer, CCT3, slc7a11, ferroptosis, EGFR

Posted Date: February 22nd, 2022

DOI: https://doi.org/10.21203/rs.3.rs-1139656/v1

License: (a) (1) This work is licensed under a Creative Commons Attribution 4.0 International License. Read Full License 
Page $2 / 22$ 


\section{Abstract \\ Background}

Chaperonin containing TCP1 subunit 3 (СCT3) acts as an oncogene in cancers, whereas its role in lung cancer is poorly understood. This study investigated the clinical relevance and function of CCT3 in lung cancer.

\section{Methods}

Clinical relevance of CCT3 in lung cancer was analyzed based on TCGA database. qRT-PCR and Western blot assay were used to detect mRNA and protein expression, respectively. CCK8 and colony formation were performed to measure cell viability. Migration was detected by transwell. PI and PI/Annexin V-FITC assay kit were used to determine cell cycle and apoptosis, respectively. Luciferase activity was performed to check the whether ССT3 regulated slc7a11's transcription activity. Ferroptosis was determined by incubating the cells with ferroptosis inducer, inhibitor and apoptosis inhibitor, followed by cell viability examination.

\section{Results}

We found that ССТ3 was overexpressed in lung cancer tissues. Overexpression of ССТ3 predicted poor prognosis in the patients. Loss-of-function and gain-of-function experiments demonstrated that CCT3 promoted the proliferation and colony formation of lung cancer cells. In addition, CCT3 knockdown suppressed cell cycle progression, cell migration and induced slc7a11-mediated cell ferroptosis, but not apoptosis. We also found that CCT3 activated EGFR and AKT. Gefitinib significantly reduced the viability of CCT3 overexpressed lung cancer cells, while had minimal effect on the proliferation of control cells, suggesting that СCT3 dictates the sensitivity of lung cancer cells to EGFR inhibition.

\section{Conclusion}

Our study demonstrates that CСT3 contributes to the proliferation and growth of lung cancer cells through inhibition of ferroptosis and activation of EGFR and AKT.

\section{Introduction}

Lung cancer is the most lethal malignancy worldwide, causing approximately 160000 death per year globally [1]. More than $80 \%$ of lung cancer patients are non-small cell lung cancer (NSCLC) and lung adenocarcinoma (LAC) represents the major histologic subtype of NSCLC [2]. The five-year overall survival of NSCLC is very poor. For the past decades, genetic, transcriptional and proteomics studies have been performed to illustrate the molecular and pathological events responsible for NSCLC development. 
As a result, targeted therapy, immunotherapy, and the combination therapy have become the promising treatment strategy for this malignancy [3-5]. However, only a small percentage of the patients benefit from the therapies and most of them relapse after years. Therefore, understanding the molecular mechanisms triggering this malignancy can help us develop effective drug target to cure this deadly disease.

Chaperonin containing TCP1 subunit 3 (CCT3) belongs to TCP1 ring complex (TRiC) family, which is consist of eight subunits, including ССT1-СCT8 $[6,7]$. It has been shown that TRiC family regulates the folding of cytoskeletal proteins, such as tubulin and actin [8,9]. Upregulation of TRiC has an important effect on cell cycle progression by regulating plk1 [10]. As a subunit of TRiC family, CCT3 is a promising diagnostic biomarker for cancers and dysregulation of ССТ3 contributes to cancer progression. ССТ3 is highly expressed in hepatocellular carcinoma (HCC) tissues and promotes the tumorigenesis of HCC [11, 12]. ССТ3 is also overexpressed in gastric cancers. Knockdown of ССТ3 reduces the viability of gastric cancer cells by regulating cell cycle proteins [13]. These results suggest that ССT3 plays an important role in cancer development. Nevertheless, the significance of ССТ3 in other cancer types, including lung cancer, is largely unclear. Studies should be conducted to illustrate the prognostic and therapeutic value of CCT3 in lung cancer.

Herein, we analyzed the prognostic value of CCT3 by analyzing the expression of CCT3 in lung cancer tissues and its correlation with the prognosis of lung cancer patients. We also performed loss-of-function and gain-of-function experiments to study the role of CCT3 in lung cancer cell growth and proliferation. Our study suggests that ССТ3 acts as an oncogene in lung cancer.

\section{Results}

\section{CCT3 is upregulated in lung cancer tissues.}

To explore the role of CCT3 in lung cancer, we first analyzed CCT3 based on TCGA database. We found that CCT3 was significantly upregulated in lung adenocarcinoma tissues (Fig. 1A). Then lung cancer and paired adjacent normal tissues were collected for mRNA and protein examination of CCT3. The results showed that the transcript of СCT3 was increased in cancer tissues comparing with normal tissues (Fig. 1B). Western blot results CCT3 was upregulated at protein level in cancer tissues (Fig. 1C). Furthermore, we detected the mRNA and protein expression of CCT in normal lung cell and cancer cells. The results showed that CCT3 was highly expressed in lung cancer cells, including A549, H1299 and H1975, as compared with normal cell Beas-2B (Fig. 1D and 1E).

\section{CCT3 expression predicts the prognosis of lung cancer patients.}

Lung cancer is the most lethal malignancy worldwide. We next investigated the significance of CCT3 on the survival of lung cancer patients. Based on the data from TCGA, we found that CCT3 expression was correlated with the prognosis of lung cancer patients. Firstly, the data analysis was performed using Quartile. Patients with overexpressed СCT3 had shorter overall and disease-free survival than patients 
with lowly expressed CCT3 (Fig. 2A, $p=0.00062$ and Fig. 2B, $p=0.0068$ ). Secondly, the data analysis was performed using Median. Patients with overexpressed CCT3 had shorter overall survival than patients with lowly expressed CCT3 (Fig. 2C, $p=0.0027$ ). However, there was no significant difference when analyzing disease-free survival (Fig. 2D). These results suggest that CCT3 is associated with the progression of lung cancer.

\section{СCT3 contributes to the growth and migration of lung cancer cells.}

To examine the effect of ССT3 on lung cancer cell function, we knocked down and overexpressed CCT3 in H1299 and H1975 cells using lentivirus. qRT-PCR and Western blot results demonstrated that CCT3 was efficiently silenced and ectopically expressed in $\mathrm{H} 1299$ cells comparing with the control cells (Fig. 3A). CCK8 experiments showed that CCT3 knockdown inhibited the proliferation, while its overexpression promoted the proliferation of H1299 cells (Fig. 3B), suggesting that CCT3 contributes to lung cancer proliferation. To confirm the results, ССТ3 knockdown and overexpression were conducted in another cancer cells H1975. Likewise, CCT3 was silenced and overexpressed in $\mathrm{H} 1975$ cells as shown by qRT-PCR and Western blot results (Fig. 3C). Downregulation and upregulation of CCT3 suppressed and promoted the proliferation of $\mathrm{H} 1975$ cells, respectively (Fig. 3D). We also found that CCT3 knockdown repressed, while its overexpression enhanced the colony formation of $\mathrm{H} 1299$ and $\mathrm{H} 1975$ cells (Fig. 3E and 3F). Furthermore, CCT3 knockdown resulted in G0/G1 cell cycle arrest in H1299 and H1975 cells (Fig. 3G).

Lung cancer is a malignancy with high metastasis rate. Next, we explored the role of CCT3 in lung cancer cell migration. The results showed that СCT3 knockdown reduced migration capacity in $\mathrm{H} 1299$ cells (Fig. $3 \mathrm{H}$ ). Collectively, СCT3 contributes to the growth and migration in lung cancer cells.

\section{CCT3 regulates ferroptosis of lung cancer cells.}

To determine whether CCT3 modulates apoptosis, we subjected СCT3 knockdown, overexpressed and control cells to Annexin V-APC/PI staining. Stained cells were analyzed on flow cytometry for apoptosis detection. We found that CСT3 knockdown had no effect on cell apoptosis, but promoted necroptosis in lung cancer cells (Fig. 4A and 4B). To validate whether CCT3 regulates ferroptosis, we treated $\mathrm{H} 1299$ cells transfected with shCtrl, shCCT3 or Ctrl, ССT3 lentivirus with ferroptosis inducer erastin, with erastin and ferroptosis inhibitor ferrostatin-1, and with erastin and apoptosis inhibitor Z-VAD-FMK. Cell viability was determined 6 hours later by CCK8 assay. We found that CCT3 knockdown enhanced, while CCT3 overexpression reduced the sensitivity of $\mathrm{H} 1299$ cells to erastin treatment (Fig. 4C and 4D, p < 0.05). Induction of cell death was rescued by ferroptosis inhibitor ferrostatin-1 but not by apoptosis inhibitor ZVAD-FMK (Fig. 4C and 4D, p < 0.05), suggesting that CCT3 suppressed cell ferroptosis but not apoptosis in lung cancer cells. These results indicate that CCT3 exhibits anti-ferroptosis effect on lung cancer cells.

\section{CCT3 transcriptionally activates slc7a11 to suppress ferroptosis.}


Slc7a11 is an essential ferroptosis regulator. Based on qRT-PCR results, we showed that CCT3 silencing downregulated slc7a11 at mRNA level. Immunoblotting assays indicated that CCT3 knockdown reduced slc7a11's protein expression (Fig. 5A). By contrast, CCT3 overexpression upregulated slc7a11 at both mRNA and protein level in $\mathrm{H} 1299$ and $\mathrm{H} 1975$ cells (Fig. 5B). These results suggested that CCT3 positively regulated the expression of slc7a11 at transcriptional manner. To address this question, we performed dual luciferase activity assay when interfering or overexpressing ССТ3. We showed that ССТ3 knockdown reduced, while the overexpression enhanced the luciferase activity of slc7a11 promoter (Fig. 5C). Importantly, CCT3 was positively correlated slc7a11 in lung cancer patients. Overexpression of slc7a11 predicted poor prognosis of the patients (Fig. 5D). To validate the role of slc7a11 in lung cancer growth and ferroptosis, slc7a11 was silenced by siRNAs transfection in $\mathrm{H} 1299$ and H1975 cells. As expected, slc7a11 downregulation inhibited the growth of both cells (Fig. 5E and 5F). Furthermore, slc7a11 knockdown resulted in increased ferroptosis in the cells (Fig. 5G). Therefore, CCT3 upregulation of slc7a11 suppresses the ferroptosis and promotes the proliferation of lung cancer cells.

\section{CCT3 activates EGFR/AKT signaling pathway in lung cancer cells.}

EGFR/AKT signaling pathway is hyper-active in various cancers, including lung cancer. We subjected CCT3 knockdown, overexpression and control cells to Western blot analysis of EGFR and AKT activity. The results showed that knockdown of CCT3 suppressed the phosphorylation, while had no effect on the expression of EGFR and AKT in H1299 cells (Fig. 6A). By contrast, CCT3 overexpression activated EGFR and AKT in H1299 cells (Fig. 6A). Furthermore, we used gefitinib, a specific EGFR inhibitor, to treat H1299 cells without or with CCT3 overexpression. We found that gefitinib obviously inhibited the proliferation and migration in CCT3 overexpressed, but not control lung cancer cells (Fig. 6B and 6C). These results suggest that CCT3 activates EGFR/AKT signaling pathway in lung cancer cells.

Overall, CCT3 promotes lung cancer cell growth through inhibition of slc7a11-mediated ferroptosis and activation of EGFR/AKT signaling pathway (Fig. 6D).

\section{Discussion}

In this present study, we investigate the clinical relevance and function of ССT3 in lung cancer. We found that ССТ3 was highly expressed in lung cancer tissues. Overexpression of ССТ3 predicted poorer overall and disease-free survival of lung cancer patients than ССТ3 low expression. Functional experiments showed that ССТ3 expression was critical for the proliferation and growth of lung cancer cells. Apoptosis was suppressed by CCT3. Furthermore, CCT3 activated EGFR/AKT signaling. EGFR specific inhibitor gefitinib suppressed the proliferation and migration of lung cancer cells. Therefore, we reveal that CCT3 promotes lung cancer cell proliferation and growth through positively regulating EGFR/AKT signaling cascade.

As a subunit of TRiC family, CCT3 plays a pivotal role in cancer progression. CCT3 is overexpressed in the tissues and serum of HCC patients. Upregulation of CCT3 promotes the development of HCC by regulating cell cycle and DNA replication pathways $[11,12]$. In gastric cancer, CCT3 is highly expressed in 
the cancer tissues comparing with normal tissues. CCT3 augments the viability of gastric cancer cells by modulating cell cycle proteins, including mitogen-activated protein kinase 7, cyclin D3, and cyclindependent kinases [13]. In addition, CCT3 is also overexpressed in papillary thyroid carcinoma (PTC) specimens. Knockdown of CCT3 significantly suppresses the proliferation and cell cycle progression of PTC cells [14]. A recent study demonstrated that knockdown of ССТ3 blunted the proliferation and migration of breast cancer cells by inhibiting NF-KB signaling. Overexpression of NF-KB reversed the inhibitory effect of CCT3 silencing on breast cancer cell proliferation and migration [15]. However, whether ССТ3 acts as a tumor suppressor or oncogene in lung cancer remains to be determined. In this study, we showed that CCT3 was upregulated in lung cancer tissues based on TCGA database and our qRT-PCR and immunoblotting analysis. CCT3 was also upregulated in lung cancer cells comparing with lung epithelial cells. We also showed that CCT3 expression was inversely correlated with the overall and disease-free survival of lung cancer patients. These results indicate that ССТ3 is a potential biomarker for lung cancer. Furthermore, we demonstrated that ССT3 knockdown suppressed, while ССТ3 ectopic expression promoted the proliferation and colony formation of lung cancer cells. Apoptosis of lung cancer cells was increased and decreased by СCT3 knockdown and overexpression, respectively. These results highlight the oncogenic role of ССТ3 functions in lung cancer.

In recent years, ferroptosis was reported as an important subtype of cell death during cancer development [16]. Inhibition of ferroptosis promotes carcinogenesis in different cancers, including lung cancer, breast cancer and melanoma [17-19]. As an essential ferroptosis repressor, slc7a11 was overexpressed in some cancer tissues and contributed to cancer growth. In addition, slc7a11 was regulated by various oncogenes or tumor suppressors. For example, loss-of-function of P53 inhibit ferroptosis and to promote cancer growth through upregulation of slc7a11 [20]. Slc7a11 can also be inhibited by radiotherapy or immunotherapy [21]. In this study, we identified СCT3 as a novel regulator of slc7a11. ССT3 upregulation of slc7a11 suppressed ferroptosis but not apoptosis. These results provide a newly evidence that CCT3 serves as ferroptosis suppressor through regulating the expression of slc7a11.

EGFR is hyper-active in lung cancer patients, largely due to gain-of-function mutation of EGFR and dysregulation of upstream modulators $[3,22,23]$. Activation of EGFR enhances the activity of PI3K/AKT in cancer [24]. Likewise, EGFR inhibitors, including osimertinib and gefitinib, are widely applied for the treatment of lung cancer patients with EGFR activation $[3,25,26]$. However, only a small number of the patients response to the treatment and most of them relapse. Identifying novel regulators of EGFR signaling may help us formulate EGFR inhibitor treatment for the appropriate patients. Here, we showed that CCT3 potentiated EGFR/AKT signaling cascade in lung cancer cells. Importantly, EGFR inhibitor, gefitinib, significantly suppressed the growth and migration in ССТЗ highly expressed lung cancer cells. Our study not only indicates that CCT3 activates EGFR/AKT signaling to promotes lung cancer, but also shows that ССТ 3 expression level is important to targeted treatment by EGFR inhibitors in lung cancer cells.

\section{Conclusion}


Overall, we present the evidences that СCT3 functions as an oncogene in lung cancer. CCT3 is highly expressed in lung cancer tissues and confers poor prognosis of lung cancer patients. Overexpression of CCT3 promotes the proliferation and growth of lung cancer cells. In addition, CCT3 inhibits ferroptosis through positive regulation of slc7a11. Importantly, CCT3 activates EGFR/AKT signaling cascade and EGFR inhibitor gefitinib could reverse the oncogenic role of CCT3. Therefore, targeting EGFR may be effective for lung cancer patients with highly expressed CCT3.

\section{Methods}

\section{Cell lines and reagents}

Lung epithelial cells Beas-2B and lung cancer cells A549, H1299 and H1975 were obtained from American Type Culture Collection (Manassas, VA, USA). The cells were maintained in Dulbecco's Modified Eagle Medium or RPMI 1640 Medium (Gibco, California, USA), containing 10\% fetal bovine serum (Gibco) and $1 \%$ antibiotics (Corning, New York, USA). Cell culture was conducted in a $370 \mathrm{C}$ incubator with $5 \%$ C02. EGFR inhibitor gefitinib was purchased from Selleckchem (TX, USA). Ferrostatin-1was obtained from Xcess Biosciences. Other drugs were from Sigma-Aldrich. Antibodies against p-EGFR, T-EGFR, p-AKT and T-AKT were obtained from Cell Signaling Technology (Danvers, USA). Slc7a11 primary antibody was from Abcam (Cambridge, United Kingdom). GAPDH primary antibody and all the secondary antibodies were from Proteintech (Chicago, USA).

\section{Human lung cancer tissues and The Cancer Genome Atlas}

Human lung cancer tissues and adjacent tissues were collected from the patients before any therapeutic intervention at Kunming Fourth People's Hospital, Seventh Affiliated Hospital of Dali University. Written informed consent was obtained from the patients. The experiments were approved by Ethics Committee of Kunming Fourth People's Hospital, Senventh Affiliated Hospital of Dali University (ky201620) and performed according to the World Medical Association Declaration of Helsinki. The mRNA and protein expression of СCT3 was analyzed in these tissues.

Transcript abundance of CCT3, overall survival, and disease-free survival of lung adenocarcinoma were analyzed from http://gepia.cancer-pku.cn/. For overall and disease-free survival analysis, lung adenocarcinoma patients were divided into СCT3 high $(n=120)$ and low group $(n=120)$.

\section{ССT3 knockdown and overexpression}

Lentivirus system was applied to knock down and overexpress CCT3. For CCT3 knockdown, short hairpin RNAs (shRNA) targeting CCT3 was cloned into pLK01.1-puro vector. The shRNA targeting sequence of CCT3 was as follow: 5'-GCCAAGTCCATGATCGAAATT-3'. For CCT3 overexpression, the coding sequence of CCT3 was cloned into pLV105 plasmid. To package lentivirus, psPAX2 and pLV-VSVG vectors were cotransfected with the lentivirus vectors into 293FT cells. The virus were harvested 48 hours later and used 
to infect H1299 and H1975 cells. Puromycin was used to construct stable cell lines with CCT3 knockdown and overexpression.

\section{Quantitative real-time PCR (qRT-PCR)}

Human lung cancer tissues or indicated cells were subjected to total RNA extraction using TRIzol reagent (Invitrogen, Carlsbad, CA, USA), according to the manufacturer's protocols. Then, 1 ug of the RNA was reversely transcribed into cDNA using RT-for-PCR kit (Clontech, Takara, Japan). Quantification of mRNA level was performed using SYBR Green II (Takara, Japan). The primer sequence were as follow: CCT3 forward, 5'-TCAGTCGGTGGTCATCTTTGG-3' and reverse, 5'-CCTCCAGGTATCTTTTCCACTCT-3'; GAPDH forward, 5'-TGTGGGCATCAATGGATTTGG-3' and reverse, 5'-ACACCATGTATTCCGGGTCAAT-3'. The 2- $\triangle \triangle \mathrm{Ct}$ method was applied to determine mRNA expression and GAPDH acted as internal control.

\section{Immunoblotting}

Total protein was extracted from human tissues and cells using RIPA lysis buffer (Beyotime Biotechnology, Shanghai, China), which was supplemented with protease and phosphatase inhibitor cocktail (Roche, Basel, Switzerland). 30-50 ug of the protein was separated on SDS- polyacrylamide gel electrophoresis (SDS-PAGE) and transferred onto PVDF membranes. After blocking with $5 \%$ non-fat milk for 1 hour, the membranes were incubated with primary antibodies at $4^{\circ} \mathrm{C}$ overnight and with secondary antibodies at room temperature for 3 hours. Protein abundance was detected by Chemiluminescent ECL reagent (Beyotime Biotechnology). Antibody against CCT3 (60264-1-lg), GAPDH (60004-1-lg) and $\beta$-actin (66009-1-lg) were obtained from Proteintech. Slc7a11 antibody (PA1-16893) was from Invitrogen. p-EGFR (\#3777), EGFR (\#4267), p-AKT (\#4060) and AKT (\#4691) primary antibodies were purchased from Cell Signaling Technology. Mouse (SA00001-1) and rabbit (SA00001-2) secondary antibodies were from Proteintech.

\section{Cell proliferation and ferroptosis detection}

CCK8 kit (YEASEN, Shanghai, China) was used to detect cell proliferation. Indicated lung cancer cells were seeded into 96 -well plates in triplicate at the density of 2000 cells per well. $6,30,54$ and 78 hours later, the OD value at $450 \mathrm{~nm}$ was detected. Cell viability at 6 hours after seeding was recognized as day 1. Cell proliferation was normalized to the OD450 value of day 1.

To determine ferroptosis, we treated the cells with ferroptosis inducer erastin, with erastin and ferroptosis inhibitor ferrostatin-1, and with erastin and apoptosis inhibitor Z-VAD-FMK. Then cell viability was examined.

\section{Colony formation assay}

For CCT3 knockdown, a total of 2000 shCtrl and shCCT3 H1299 and H1975 cells were seeded into 6-well plates. For CCT3 overexpression, a total of $800 \mathrm{Ctrl}$ and CCT3 overexpressed H1299 and H1975 cells were 
seeded. 10 days later, colonies were washed with PBS and stained with crystal violet solution. Images of colonies were photographed under the camera.

\section{Apoptosis and cell cycle detection}

Apoptosis of lung cancer cells was measured using PI/Annexin V-FITC kit (Invitrogen). In brief, control, CCT3 silenced and CCT3 overexpressed H1299 and H1975 cells in 6-well plates in triplicate were trypsinized and washed by PBS. After stained with PI and Annexin V-FITC, apoptosis was detected on the flow cytometer (BECKMAN COULTER, Minnesota, USA), according to the manufacturer's protocols.

Cell cycle of lung cancer cells was measured using PI kit (YEASEN, Shanghai, China) and was detected on the flow cytometer (BECKMAN COULTER), according to the manufacturer's protocols.

\section{Transwell assay}

Equal amount of H1299 or H1975 cells were seeded into the upper surface of transwell chamber. Upper chamber contained 200 ul FBS-free culture medium. 500 ul culture medium containing 10\% FBS was seeded into the 24-well plates. 24 hours later, cells attached on the upper surface were removed by cotton bud. Cell attached on the lower surface were washed by PBS and fixed by methanol. After stained by crystal violet and washed by clean water, the chambers were dried at room temperature. Microscope was used to collect pictures of migrated cells.

\section{Dual luciferase reporter assay}

The promoter sequence was cloned into pGL3.basic vector. Coding sequence of CCT3 was inserted into pCDNA3.1. After transfecting pCDNA3.1, pGL3.basic and TK vectors to $\mathrm{H} 1299$ cells, dual luciferase activity was assessed by using Dual-Luciferase Reporter Assay Kit (Promega, USA), according to manufacturers' instructions. The luciferase activity was adjusted to Renilla luciferase expression vector, pCMV-RL-TK.

\section{Statistical analysis}

All the data were presented as mean \pm standard error of the mean (SEM) and were analyzed using GraphPad Prism. Student's t test was used to compare the difference between two groups. One-way ANOVA was applied when more than two groups. Statistical difference was considered significantly when $P$ was less than 0.05 .

\section{Declarations}

\section{Data Availability Statement}

All of the data generated during this study were included in this article.

\section{Acknowledgements}


Not applicable.

\section{Funding}

This work was supported by Kunming Municipal Health Committee Health Research Project (2020-04-02115) and Science Research Fund Project of Yunnan Provincial Department of Education (2018JS226).

\section{Author information}

Kun Wang and Jian He contributed equally to this work.

\section{Affiliations}

\section{Yunnan Kungang Hospital, Kunming Fourth People's Hospital, Seventh Affiliated Hospital of Dali University}

Kun Wang, Jian He, Hui Xu, Xugang Zhang, Yongchang Lv, Chao Song

\section{The Third Affiliated Hospital of Kunming Medical University}

Changling Tu

\section{Contributions}

K.W., J.H. and C.S. designed this study. K.W., J.H. and C.T. conducted the experiments. K.W., J.H., C.S., H.X., X.Z. and Y.L. analyzed the data. K.W., J.H. and C.S. wrote the manuscript. The authors have read and approved the final manuscript.

\section{Corresponding author}

Chao Song

\section{Ethics approval and consent to participate}

This study was approved by the Ethics Committee of Kunming Fourth People's Hospital, Senventh Affiliated Hospital of Dali University. Written informed consent was obtained from the patients. The experiments were performed according to the World Medical Association Declaration of Helsinki

\section{Consent for publication}

Not applicable.

\section{Competing interests}

The authors declare no conflicts of interest. 


\section{References}

1. Torre LA, Bray FI, Siegel RL, Ferlay J, Lortettieulent J, Jemal A: Global cancer statistics, 2012. CA Cancer J Clin 2015, 65(2):87-108.

2. S D, D M, R G: Genomic alterations in lung adenocarcinoma. The Lancet Oncology 2015, 16(7):e342351.

3. AJ P-V, LV S, Z P: Emerging Treatment Paradigms for EGFR-Mutant Lung Cancers Progressing on Osimertinib: A Review. Journal of clinical oncology : official journal of the American Society of Clinical Oncology 2020:JC01903123.

4. D A, S O-E, H M, T L, MJ P, J A, C B, JJ L, S V, K H et al: A Combined PD-1/C5a Blockade Synergistically Protects against Lung Cancer Growth and Metastasis. Cancer Discov 2017, 7(7):694703.

5. J B, KL R, P B, L C, WE E, E P, S A, A P, EE V, E H et al: Nivolumab versus Docetaxel in Advanced Squamous-Cell Non-Small-Cell Lung Cancer. The New England journal of medicine 2015, 373(2):123-135.

6. V S, F R, V K, JF E, DA P, F S: Review: the Cct eukaryotic chaperonin subunits of Saccharomyces cerevisiae and other yeasts. Yeast (Chichester, England) 1996, 12(6):523-529.

7. JM V, J M-B, P G-P, JL C, KR W: Structure and function of a protein folding machine: the eukaryotic cytosolic chaperonin CCT. FEBS Lett 2002, 529(1):11-16.

8. MB Y, GW F, D M, AL H, ML S, H S: TCP1 complex is a molecular chaperone in tubulin biogenesis. Nature 1992, 358(6383):245-248.

9. H S, GW F, ML S, JK D, K W, MB Y: The t-complex polypeptide 1 complex is a chaperonin for tubulin and actin in vivo. Proc Natl Acad Sci U S A 1993, 90(20):9422-9426.

10. Liu X, Lin CY, Lei M, Yan S, Zhou T, Erikson RL: CCT chaperonin complex is required for the biogenesis of functional Plk1. Mol Cell Biol 2005, 25(12):4993-5010.

11. EN Q, SY H, SZ D, X L: Expression and diagnostic value of CCT3 and IQGAP3 in hepatocellular carcinoma. Cancer Cell Int 2016, 16:55.

12. JY H, HY W, RQ H, P L, YW D, G C: Clinical and prognostic value of chaperonin containing T-complex 1 subunit 3 in hepatocellular carcinoma: A Study based on microarray and RNA-sequencing with 4272 cases. Pathol Res Pract 2019, 215(1):177-194.

13. LJ L, LS Z, ZJ H, ZY H, H C, YM L: Chaperonin containing TCP-1 subunit 3 is critical for gastric cancer growth. Oncotarget 2017, 8(67):111470-111481.

14. X S, S C, W W: CCT3Suppression of inhibits malignant proliferation of human papillary thyroid carcinoma cell. Oncol Lett 2018, 15(6):9202-9208.

15. G X, S B, X W, H Z, H G: Suppression of CCT3 inhibits the proliferation and migration in breast cancer cells. Cancer Cell Int 2020, 20:218.

16. Zheng J, Conrad M: The Metabolic Underpinnings of Ferroptosis. Cell Metab 2020, 32(6):920-937. 
17. Bersuker K, Hendricks JM, Li Z, Magtanong L, Ford B, Tang PH, Roberts MA, Tong B, Maimone TJ, Zoncu R et al: The CoQ oxidoreductase FSP1 acts parallel to GPX4 to inhibit ferroptosis. Nature 2019, 575(7784):688-692.

18. Ding Y, Chen X, Liu C, Ge W, Wang Q, Hao X, Wang M, Chen Y, Zhang Q: Identification of a small molecule as inducer of ferroptosis and apoptosis through ubiquitination of GPX4 in triple negative breast cancer cells. $J$ Hematol Oncol 2021, 14(1):19.

19. Yang Y, Luo M, Zhang K, Zhang J, Gao T, Connell DO, Yao F, Mu C, Cai B, Shang Y et al: Nedd4 ubiquitylates VDAC2/3 to suppress erastin-induced ferroptosis in melanoma. Nat Commun 2020, 11(1):433.

20. Jiang L, Kon N, Li T, Wang SJ, Su T, Hibshoosh H, Baer R, Gu W: Ferroptosis as a p53-mediated activity during tumour suppression. Nature 2015, 520(7545):57-62.

21. Lang X, Green MD, Wang W, Yu J, Choi JE, Jiang L, Liao P, Zhou J, Zhang Q, Dow A et al: Radiotherapy and Immunotherapy Promote Tumoral Lipid Oxidation and Ferroptosis via Synergistic Repression of SLC7A11. Cancer Discov 2019, 9(12):1673-1685.

22. K S, T M, Y S, J O, H I, T O, S T, T M, SI W, H A et al: Clinical impacts of EGFR mutation status: analysis of 5,780 surgically resected lung cancer cases. The Annals of thoracic surgery 2020.

23. C Z, C C, F Y, W F: ZNF251 promotes the progression of lung cancer by activating ERK signaling. Cancer Sci 2020.

24. F G, S Q, S G, G M, L J, MA A, LB D, LA P: Investigation of EGFR/pi3k/Akt signaling pathway in seminomas. Biotechnic \& histochemistry : official publication of the Biological Stain Commission 2020:1-13.

25. YL W, Y C, J Z, S L, Y Z, J Z, DW K, RA S, SW K, H P et al: Tepotinib plus gefitinib in patients with EGFR-mutant non-small-cell lung cancer with MET overexpression or MET amplification and acquired resistance to previous EGFR inhibitor (INSIGHT study): an open-label, phase 1b/2, multicentre, randomised trial. The Lancet Respiratory medicine 2020.

26. HA Y, AJ S, A M, R K, H R, D T, C F, B H-L, F M, JL Y et al: Effect of Osimertinib and Bevacizumab on Progression-Free Survival for Patients With Metastatic EGFR-Mutant Lung Cancers: A Phase 1/2 Single-Group Open-Label Trial. JAMA oncology 2020.

\section{Figures}


A

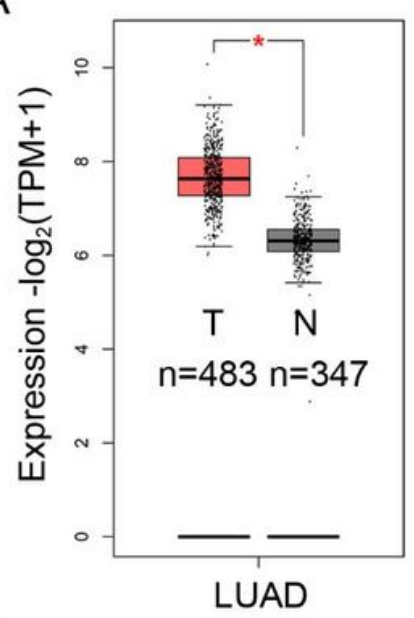

D

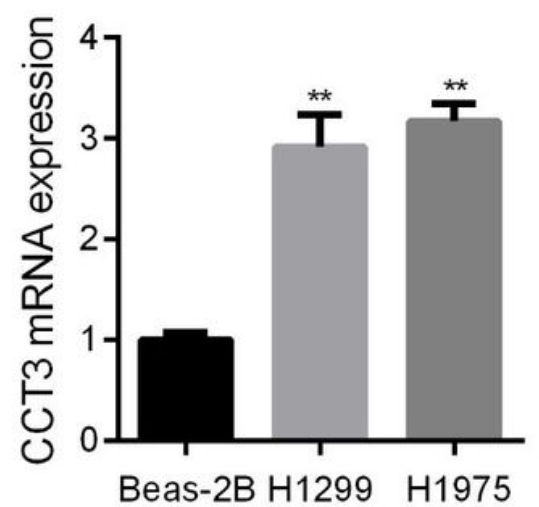

B

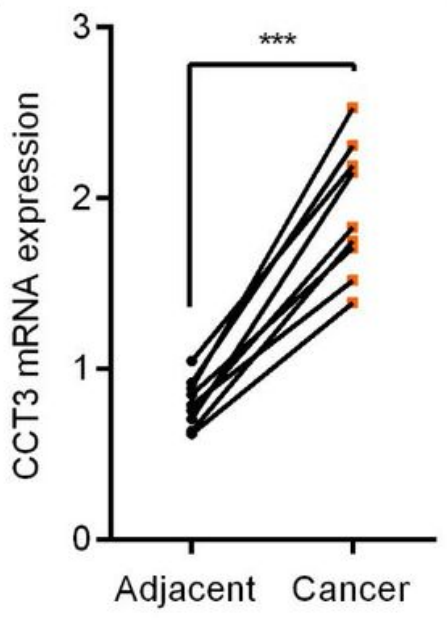

C

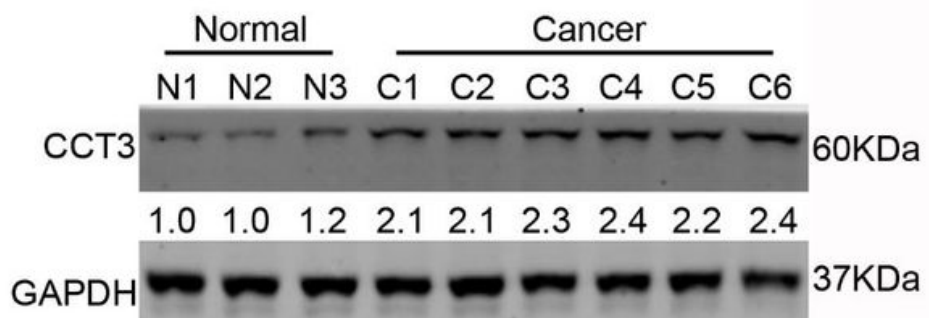

E

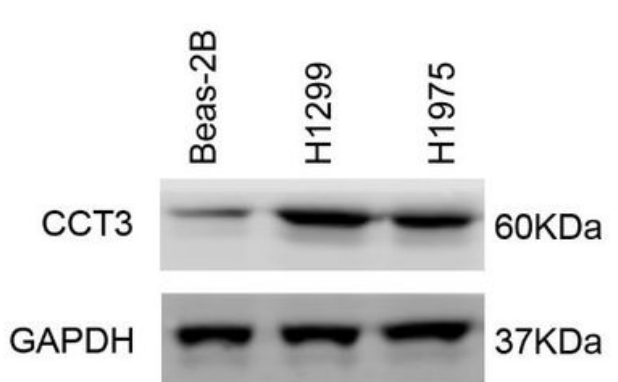

\section{Figure 1}

ССТ3 is overexpressed in lung cancer tissues and cells.

(A) The transcript abundance of ССТ3 was analyzed in lung adenocarcinoma $(n=483)$ and normal tissues ( $n=347)$ from TCGA database. ${ }^{*} p<0.05$. (B) Nine pairs of lung cancer and adjacent normal tissues were subjected to qRT-PCR analysis of CCT3 expression. ${ }^{\star \star \star} p<0.001$. (C) Western blot analysis of CCT3 in lung cancer and paired adjacent normal tissues. (D and E) qRT-PCR (D) and Western blot (E) analysis of CCT3 in normal lung cells Beas-2B and in cancer cells H1299 and H1975. ${ }^{* \star} p<0.01$. GAPDH is the internal control. 
A

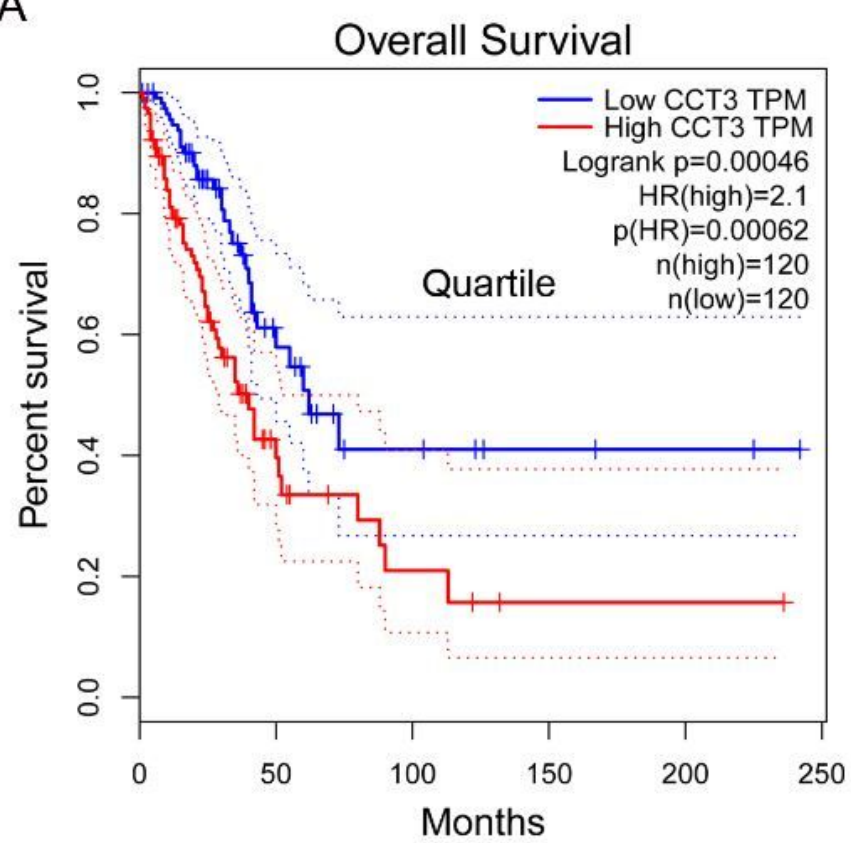

C

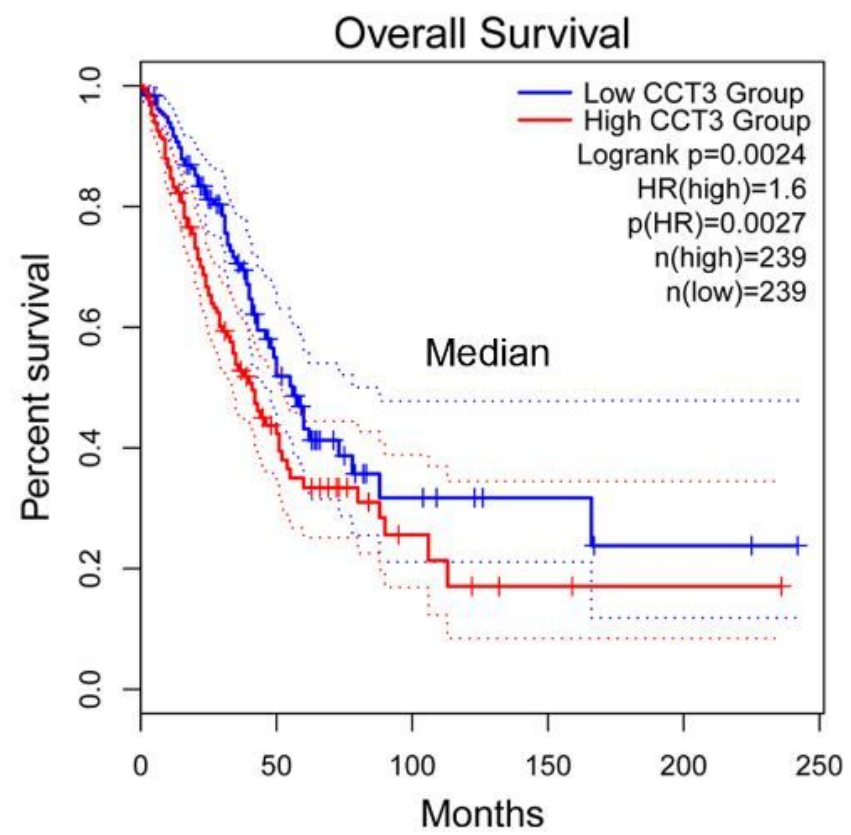

B

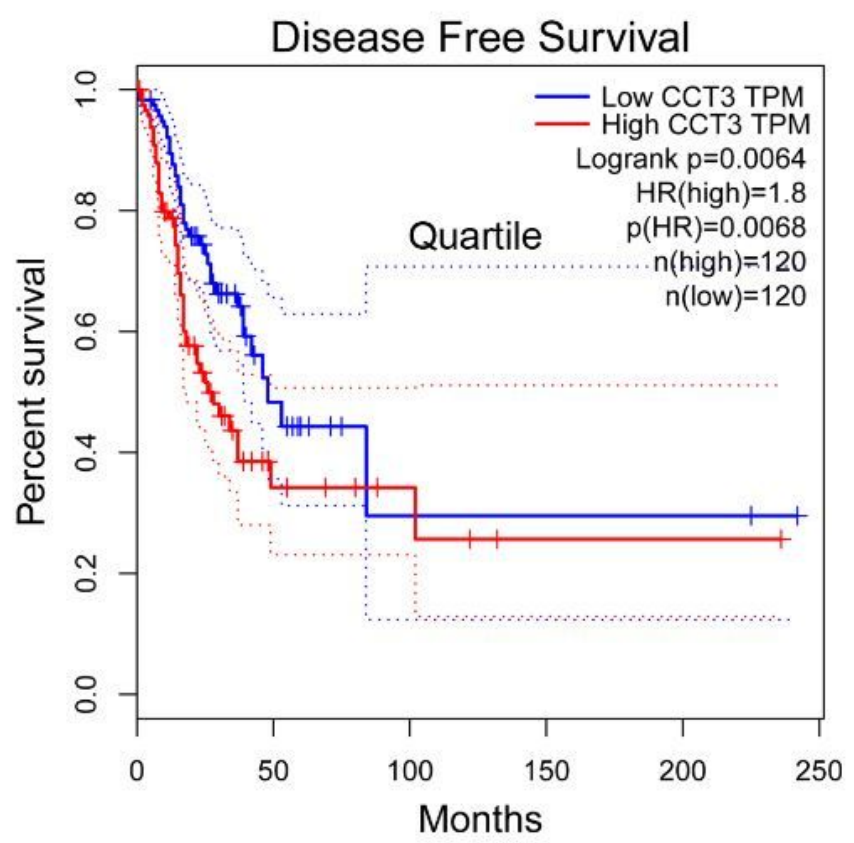

D

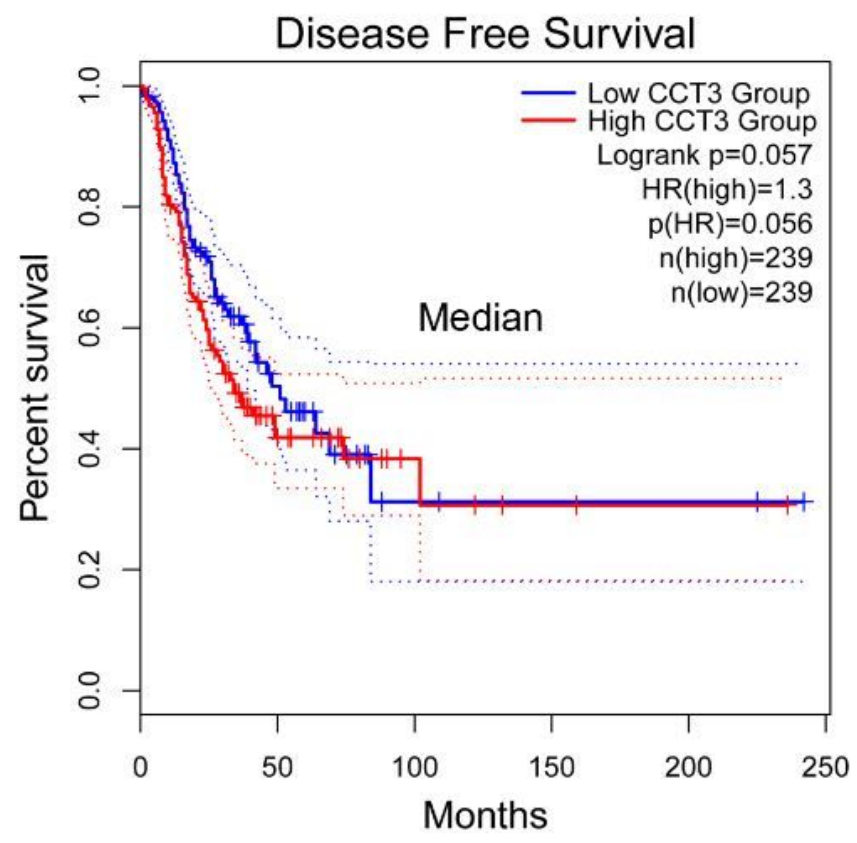

Figure 2

\section{ССТЗ expression is associated with the survival of lung cancer patients.}

(A) Overall survival of lung cancer patients with high expression and low expression of CCT3. $n=120$ in each group. $p=0.00046$. (B) Disease free survival of lung cancer patients with high expression and low expression of ССТ3. $n=120$ in each group. $p=0.0064$. The data analysis was performed using Quartile. (C) Overall survival of lung cancer patients with high expression and low expression of CCT3. $n=239$ in each group. $p=0.0027$. (D) Disease free survival of lung cancer patients with high expression and low expression of ССТ3. $n=239$ in each group. $p=0.057$. The data analysis was performed using Median. 
A
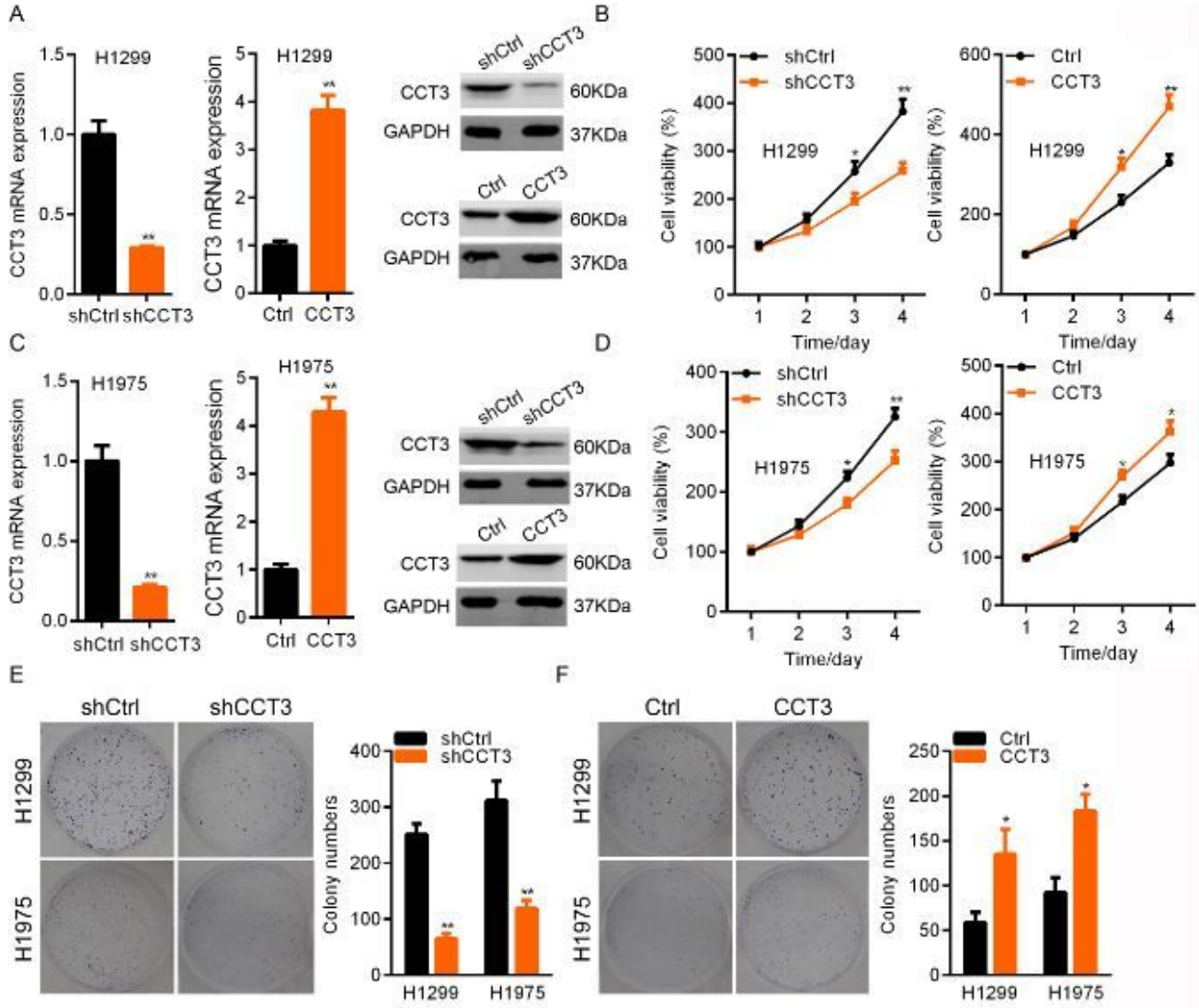

G
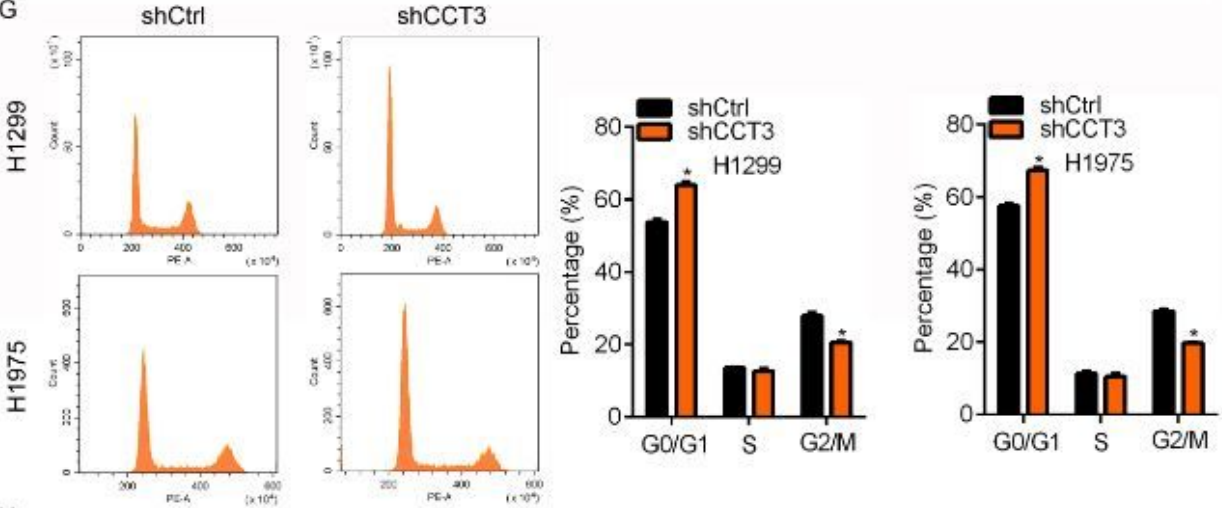

H
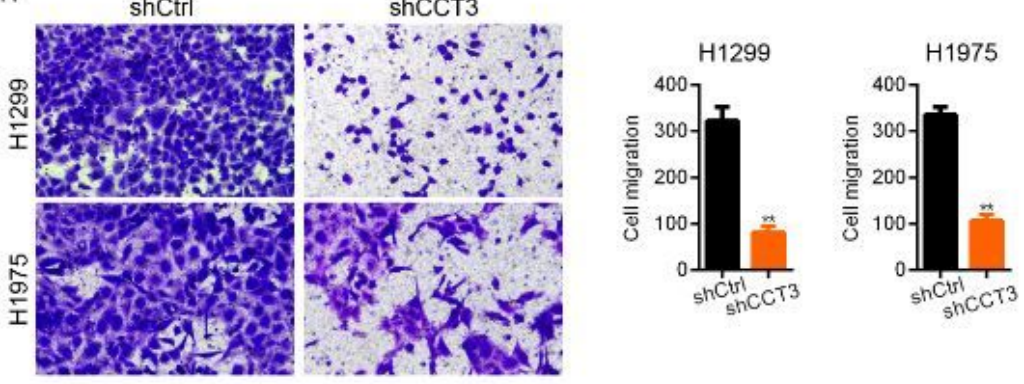

Figure 3

ССТ3 promotes the growth and migration of lung cancer cells.

(A) H1299 cells were infected with shCtrl and shCCT3 knockdown lentivirus, or infected with Ctrl and CCT3 overexpression lentivirus. qRT-PCR and Western blot analysis of CCT3 in shCtrl and shCCT3, and Ctrl and CCT3 overexpressed H1299 cells. GAPDH is the internal control. **p<0.01. (B) CCT3 knockdown, 
overexpressed and the negative control $\mathrm{H} 1299$ cells were subjected to CCK8 analysis of cell proliferation. ${ }^{*} p<0.05$. ${ }^{*} p<0.01$. (C) H1975 cells were infected with shCtrl and shCCT3 knockdown lentivirus, or infected with Ctrl and CCT3 overexpression lentivirus. qRT-PCR and Western blot analysis of CCT3 in shCtrl and shCCT3, and Ctrl and CCT3 overexpressed H1975 cells. GAPDH is the internal control. ${ }^{\star *} \mathrm{p}<0.01$. (D) CCT3 knockdown, overexpressed and the negative control $\mathrm{H} 1975$ cells were subjected to CCK8 analysis of cell proliferation. ${ }^{*} p<0.05 .{ }^{*} p<0.01$. (E) Colony formation analysis was performed on shCtrl and shCCT3 H1299 and H1975 cells. ${ }^{* *} \mathrm{p}<0.01$. (F) Colony formation analysis was performed on Ctrl and CCT3 overexpressed H1299 and H1975 cells. ${ }^{*} \mathrm{p}<0.05$. (G) Cell cycle was analyzed by PI staining in shCtrl and shCCT3 H1299 and H1975 cells. Left, representative images of cell cycle. Right, quantification of cell cycle. ${ }^{*} p<0.05$. $(\mathrm{H})$ Cell migration was determined by transwell in shCtrl and shCCT3 H1299 and H1975 cells. Left, representative images of cell migration. Right, quantification of cell migration. ${ }^{* *} \mathrm{p}<0.01$. 
A
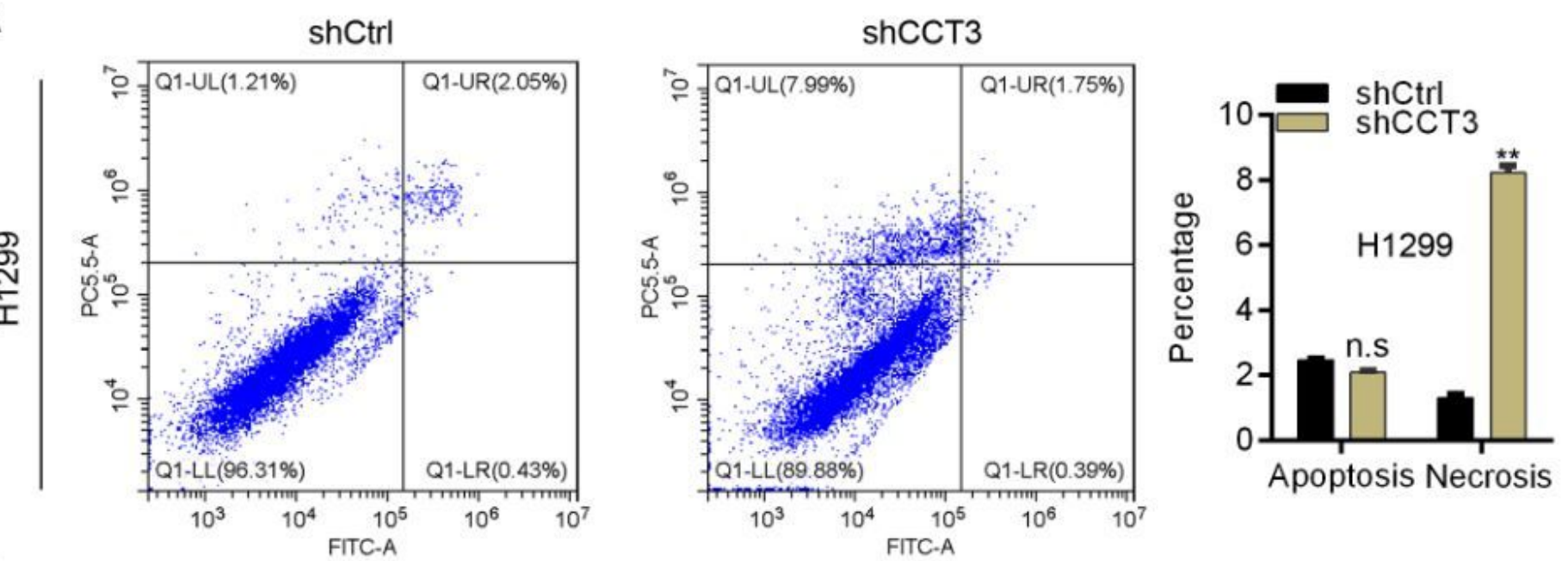

B
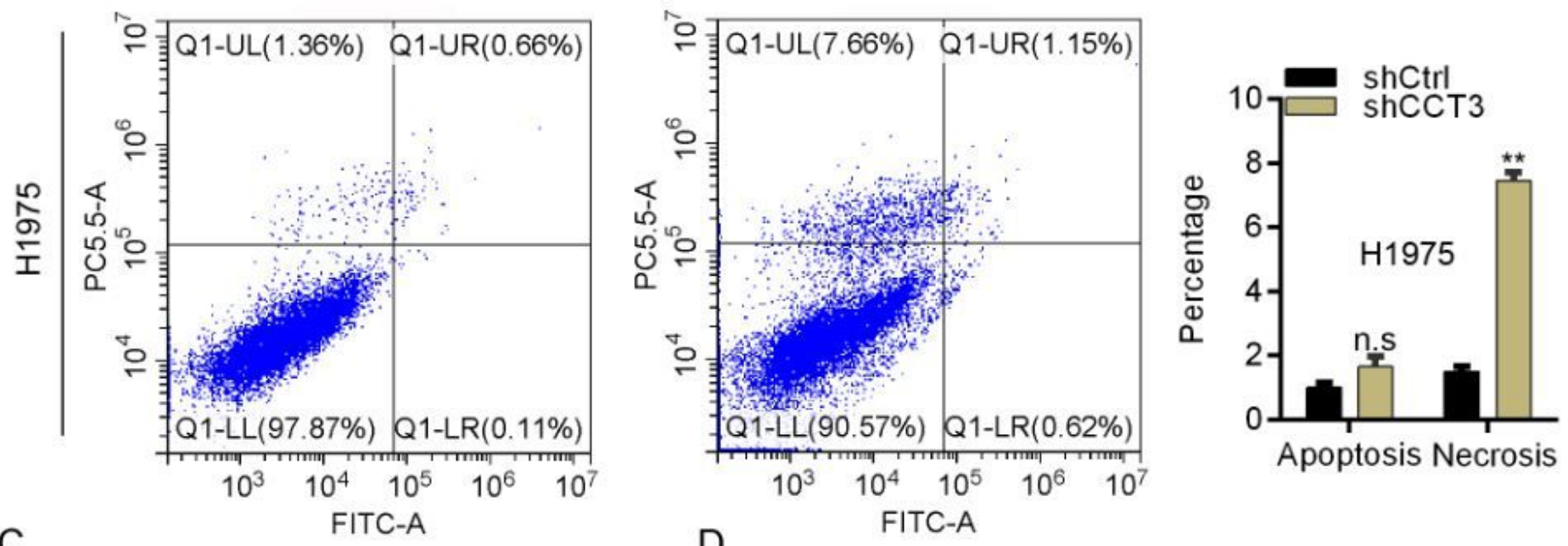

C

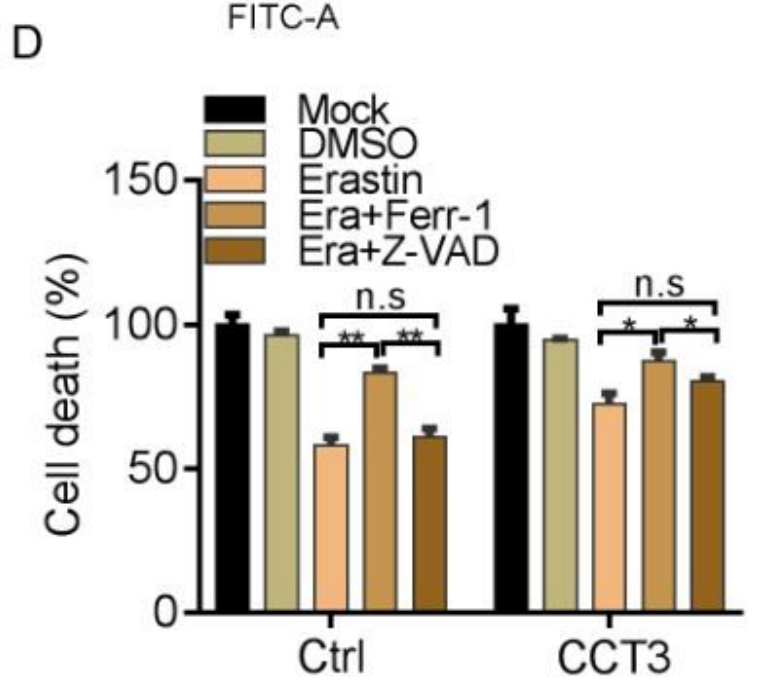

Figure 4

\section{ССT3 inhibits ferroptosis of lung cancer cells.}

(A and B) Apoptosis and necroptosis were detected by using Annexin V-APC/PI staining in shCtrl and shCCT3 H1299 (A) and H1975 (B) cells. Left, representative images of apoptosis and necroptosis. Right, quantification results. ${ }^{\star \star} \mathrm{p}<0.01$. (C and D) shCtrl and shCCT3 (C), Ctrl and CCT3 overexpressing (D) 
H1299 cells were treated with MOCK, DMSO, erastin, erastin + ferrostatin-1 (Era+Ferr-1), erastin+ Z-VADFMK (Era+Z-VAD). Cell viability was detected by CCK8 assay. n.s, not significant. ${ }^{*} p<0.05 .{ }^{* *} p<0.01$.

A

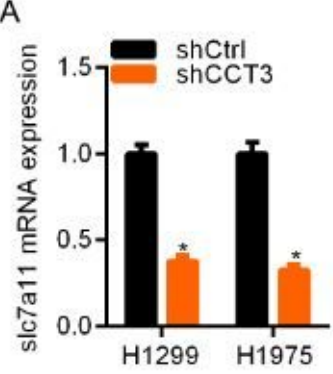

C

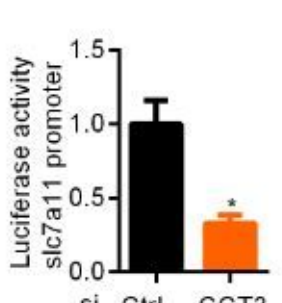

si $\mathrm{Ctrl}$ ССT3

E

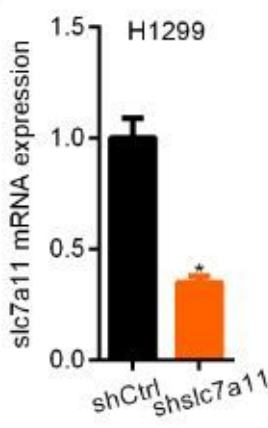

G
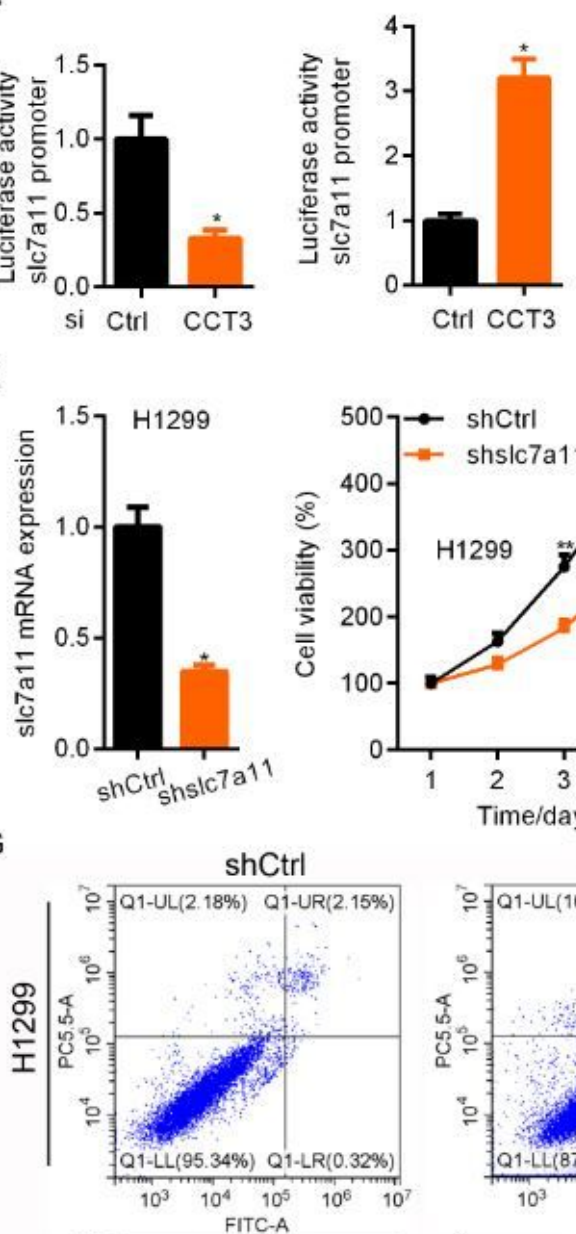

D

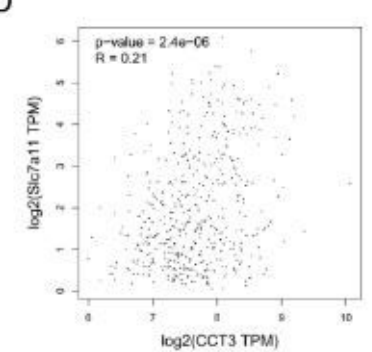

$\mathrm{F}$
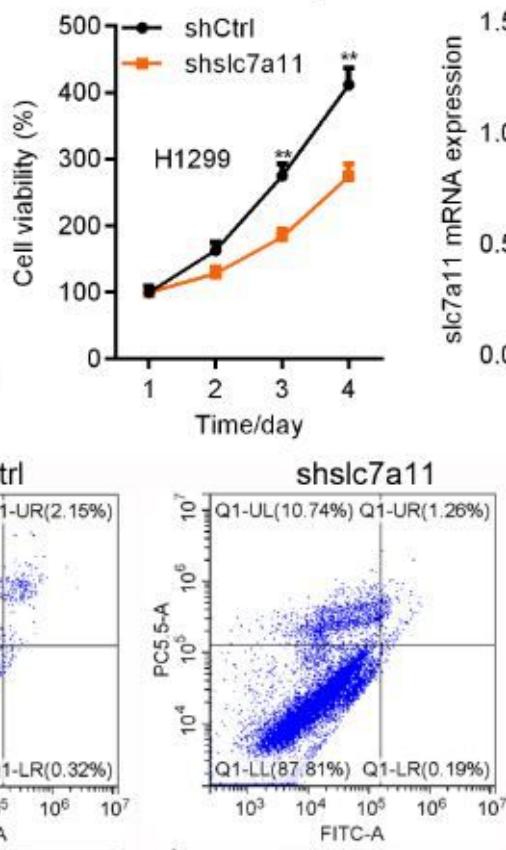

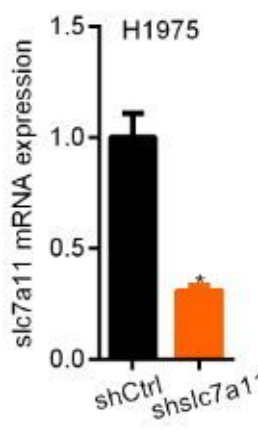

B
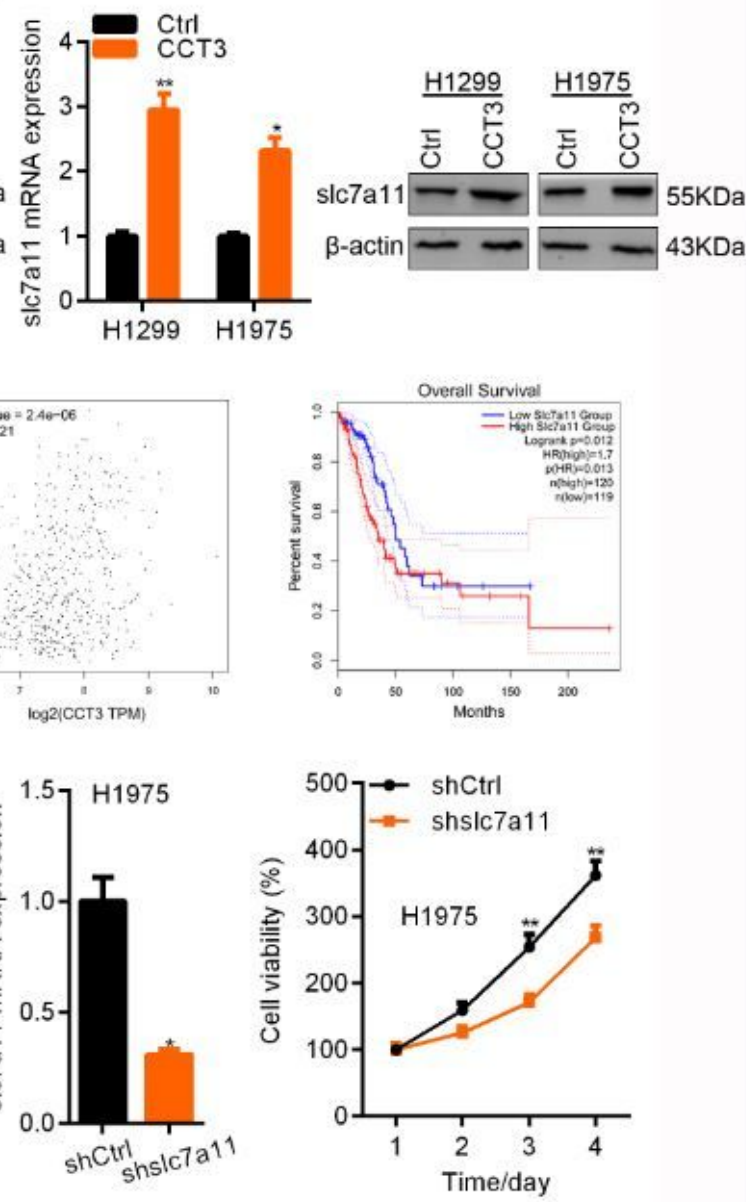
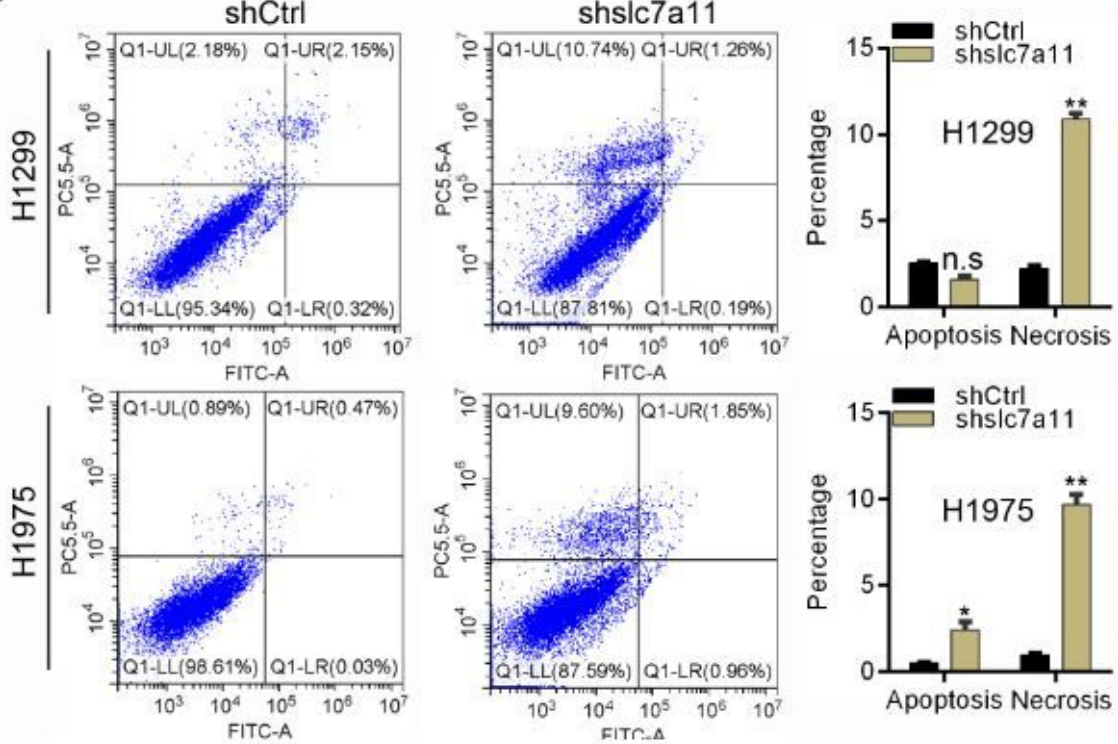

Figure 5

ССТ3 upregulation of slc7a11 inhibits ferroptosis. 
(A) qRT-PCR and Western blot analysis of slc7a11 in shCtrl and shCCT3 H1299 and H1975 cells. $\beta$-actin is the internal control. ${ }^{*} p<0.05$. (B) qRT-PCR and Western blot analysis slc7a11 in Ctrl and CCT3 overexpressed $\mathrm{H} 1299$ and $\mathrm{H} 1975$ cells. $\beta$-actin is the internal control. ${ }^{*} \mathrm{p}<0.05$. ${ }^{\star \star} \mathrm{p}<0.01$. (C) Dual luciferase activity was checked in $\mathrm{H} 1299$ cells transfected with siRNAs (siCtrl or siCCT3) or pCDNA3.1 plasmids (pCDNA3.1-Ctrl or pCDNA3.1-CCT3), pGL3.Basic-slc7a11 promoter and TK. (D) Sperman correlation between CCT3 and slc7a11 in lung cancer patients was analyzed from TCGA. Overall survival of lung cancer patients (slc7a11 high and low expression groups) was analyzed from TCGA. (E and F) qRT-PCR analysis of slc7a11 and CCK8 analysis of cell proliferation were performed in shCtrl and shslc7a11 H1299 and H1975 cells. **p<0.01. (G) Apoptosis and necroptosis were detected by using Annexin V-APC/PI staining in shCtrl and shslc7a11 H1299 and H1975 cells. Left, representative images of apoptosis and necroptosis. Right, quantification results. ${ }^{*} p<0.05 .{ }^{* \star} p<0.01$. 
A
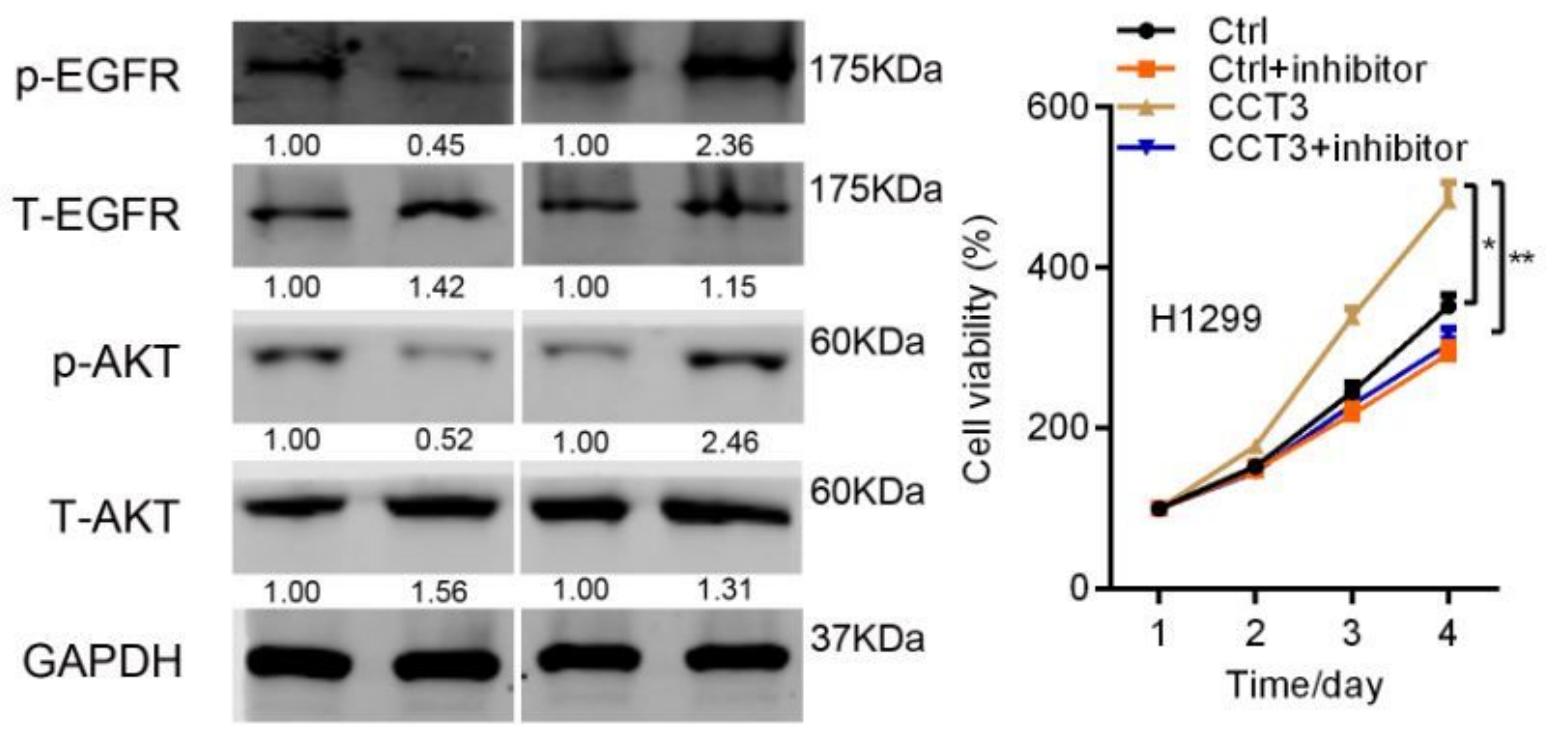

C
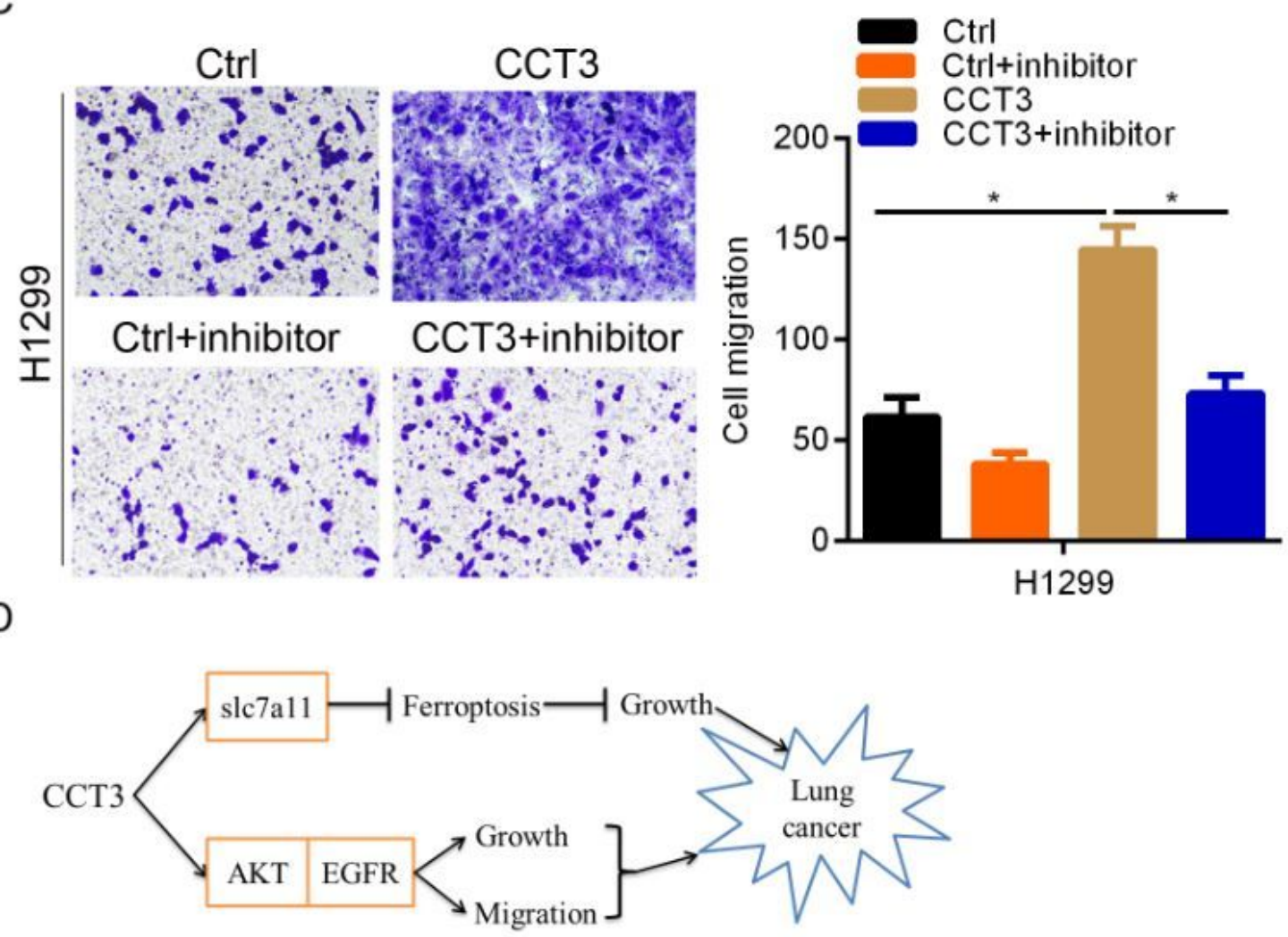

Figure 6

\section{CCT3 stimulates EGFR/AKT signaling cascade.}

(A) shCtrl and shCCT3, and Ctrl and CCT3 overexpressed H1299 cells were subjected Western blot analysis with indicated antibodies. (B) Cell viability was the determined by CCK8 assay in Ctrl, CCT3 overexpressed, Ctrl and CCT3 overexpressed H1299 cells that were treated with gefitinib for indicated 
time. ${ }^{*} p<0.05 .{ }^{*} p<0.01$. (C) Cell migration was detected by transwell assay in Ctrl, CCT3 overexpressed, $\mathrm{Ctrl}$ and CCT3 overexpressed H1299 cells that were treated with gefitinib. ${ }^{*} \mathrm{p}<0.05$. (D) Schematic diagram of how CCT3 contributes to lung cancer cell growth.

\section{Supplementary Files}

This is a list of supplementary files associated with this preprint. Click to download.

- OriginalWBbands.docx 